Review Article

\title{
Challenges of the Modeling Methods for Investigating the Interaction between the CNT and the Surrounding Polymer
}

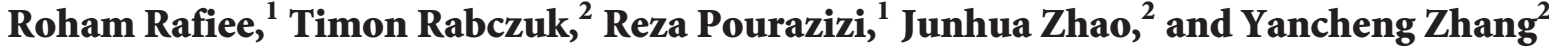 \\ ${ }^{1}$ Composites Research Laboratory, Faculty of New Sciences and Technologies, University of Tehran, Tehran 1439955941, Iran \\ ${ }^{2}$ Institute of Structural Mechanics, Bauhaus University Weimar, Marienstraße 15, 99423 Weimar, Germany \\ Correspondence should be addressed to Roham Rafiee; roham.rafiee@ut.ac.ir
}

Received 1 January 2013; Revised 27 April 2013; Accepted 3 June 2013

Academic Editor: Philip Harrison

Copyright (c) 2013 Roham Rafiee et al. This is an open access article distributed under the Creative Commons Attribution License, which permits unrestricted use, distribution, and reproduction in any medium, provided the original work is properly cited.

\begin{abstract}
The interaction between the carbon nanotubes (CNT) and the polymer is a key factor for determining the mechanical, thermal, and electrical properties of the CNT/polymer nanocomposite. However, it is difficult to measure experimentally the interfacial bonding properties between the CNT and the surrounding polymer. Therefore, computational modeling is used to predict the interaction properties. Different scale models, from atomistic to continuum, are critically reviewed addressing the advantages, the disadvantages, and the future challenges. Various methods of improvement for measuring the interaction properties are described. Finally, it is concluded that the semicontinuum modeling may be the best candidate for modeling the interaction between the CNT and the polymer.
\end{abstract}

\section{Introduction}

Predicting the mechanical properties and modeling the mechanical behavior of the CNT Reinforced Polymer (CNTRPs) have attracted the great attention of many researchers [1-5], especially considering their rapid development in various industries such as automotive industry, aerospace, packaging, and wind turbines. Therefore, over the past two decades, extensive studies have been executed to predict the mechanical properties of CNTRPs.

Different factors affect the performance of CNTRPs. The main governing issue which characterizes the efficiency of CNT reinforcement at nano-/microscale can be attributed to the load transfer mechanism from the matrix to the CNTs. As a result, prior to understanding the mechanical behavior of CNTRP at macrolevel, the corresponding physical performance has to be addressed. For this purpose, a proper simulation technique, which captures the required details sufficiently and reasonably, needs to be employed in accordance with the investigated scale.

Interfacial bonding in the interphase region between the embedded CNT and its surrounding polymer is a crucial issue for the load transferring and reinforcement phenomena. The atomic structure of carbon nanotubes consisting of $\mathrm{sp}^{2}$ hybridized carbons hinders the formation of strong covalent bonds with the surrounding polymer matrix. Although functionalization can improve the load transfer of the interphase by providing covalent cross-links between the carbon atoms of CNTs and the molecules of polymer, this procedure has a main drawback in providing defects in the structure of carbon nanotubes due to formation of $\mathrm{sp}^{3}$ hybridized sites. It can significantly reduce the beneficial properties of carbon nanotubes. Therefore, CNTs naturally interact with the polymer chains of the matrix just through weakly nonbonded van der Waals (vdW) and electrostatic forces. Mechanical interlocking, which is useful to improve the adhesion between the fiber and the matrix in fibrous composites, is not pertinent to CNTRPs due to the smooth surface of the CNTs.

Up to now, various methods have been employed to investigate the interaction behavior between the CNT and the surrounding polymer, including experimental studies, atomistic modeling, and continuum modeling. Through systematically studying the shortcomings and advantages of all the available methods from the literature, the objective of this review is to find the best solution for evaluating the interaction behavior between the CNT and the polymer matrix. On one side, the accuracy must be guaranteed; on the other side, the method should be simple and have minimal computational costs. 
This paper aims to review recently developed modeling techniques to study the interaction between CNT and surrounding polymer. The organization of the paper is as follows: Section 2 is a brief explanation of challenges imposed to the experimental studies at the scale of Nano. Section 3 discusses performed theoretical studies for investigating interaction between CNT and polymer. This section includes two main groups of available modeling techniques as atomistic modeling Section 3.1 and continuum modeling Section 3.2. Molecular dynamics, coarse grain simulation, and density functional theory are reviewed in Sections 3.1.1, 3.1.2, and 3.1.3, respectively. Then, associated obstacles and shortcomings of atomistic modeling are presented in Section 3.1.4 which are the main reasons encouraging researchers to employ continuum modeling approaches. Categorized under continuum modeling group, Sections 3.2.1 and 3.2.2 present analytical and numerical modeling techniques. Finally, Section 4 concludes the review by highlighting pros and cons of available methods.

\section{Experimental Studies}

The performed experimental studies for characterizing the interaction between CNT and surrounding polymer can be divided into three main categories.

Some researchers have performed experiments on CNTpolymer bulk composites at the macroscale and observed the enhancements in mechanical properties (like elastic modulus and tensile strength) and tried to correlate the experimental results and phenomena with continuum theories like micromechanics of composites or Kelly Tyson shear lag model [1$5,32,33]$.

Other researchers have used the spectroscopy techniques. Spectroscopy is a powerful technique for characterizing the properties of the CNT. This technique includes Raman spectroscopy, Fourier Transform infrared spectroscopy (FTIR), and X-ray scattering. The main principle of this method is that by straining the $\mathrm{CNT} /$ polymer, the $\mathrm{C}-\mathrm{C}$ bonds will vibrate, and this will affect the peaks in the Raman spectrum. Therefore, Raman spectroscopy can determine the characteristics of CNTs such as the diameters and the level of dispersion in the matrix [34-38].

In situ TEM (Transmission Electron Microscopy) straining has also been used to understand the mechanics, fracture, and failure processes of the interface. In these techniques, the CNT-polymer composite (an electron-transparent thin specimen) is strained inside a TEM and simultaneously imaged to get real-time and spatially resolved $(1 \mathrm{~nm})$ information $[39,40]$.

Rahmat and Hubert [41] and also Desai and Haque [42] have conducted a comprehensive review on the experimental methods.

Generally, an experimental study at the scale of nano is a challenging task due to the following reasons:

(i) the lack of proper direct measuring techniques at the nanometer scale;

(ii) tremendous limitations on the specimen size; (iii) an uncertainty in the data obtained from indirect measurements;

(iv) inadequate preparation techniques of test specimens and the lack of control over the alignment and distribution of nanotubes.

Therefore researchers have a tendency to employ theoretical methods for predicting properties of CNT-reinforced composites. Moreover, it is not possible to measure the interfacial bonding properties between CNT and surrounding polymer through direct measurement, and this would be another source of error.

\section{Theoretical Studies}

In this category of studies, the interaction between the CNT and the surrounding polymer which plays a key factor in transferring the load from the matrix to the CNT is examined using theoretical and computer models. Up to now, the employed theoretical studies to simulate the interaction and measure the mechanical properties of the interaction are mainly divided into two major groups: atomistic modeling and continuum modeling.

3.1. Atomistic Modeling. Atomistic modeling is accomplished using molecular dynamic (MD) simulation, density functional theory (DFT), or coarse grain (CG) simulation. These techniques are widely used to resolve the relevant fracture problems on different scales.

3.1.1. Molecular Dynamics. The MD method solves Newton's equations of motion for a molecular system [43], which provides the methodology for detailed microscopic modeling on the molecular scale [44]. For the nanocomposite interaction studies, MD has shown strong potential, due to its accuracy, flexibility, and various force fields [10, 13, 45, 46]. Xu and Buehler [10] adopted the AIREBO potential to simulate the mechanical behavior of the CNT-based nanocomposite. In the work of Zhang et al. [13], the UA (united atom) potential is adopted to address the similar problem of the nanocomposites. Moreover, the coarse-grained potential is also employed to study the interaction among particle-particle and particlepolymer $[45,46]$.

Additionally, the comparable sizes of nanotubes and polymer chains make MD a great candidate to simulate nanocomposites without sacrificing the accuracy of the results, especially with the rapid development of high-performance supercomputers.

The MD simulations can also investigate the effects of various parameters and types of interaction on mechanical properties like elastic moduli, interfacial shear strength, and bonding energy.

The interface properties of CNT/polymer were widely investigated based on MD simulation from the available literatures $[6,8-10,12,13]$. Referring to the nonbonded $\mathrm{CNT} /$ polymer interaction, Chen et al. [9] performed $\mathrm{MD}$ simulation of single polymer molecular with CNT, and they found that armchair single-walled carbon nanotubes 
(SWNTs) present the strongest adhesion. Liao and Li [6] and Rahmat and Hubert [12] studied the noncovalent interaction between the CNTs and the matrix; the shear strength and the binding energy of the composite interfaces were given in their research. Wong et al. [8] found that the locally nonuniformed distribution of CNT-induced mechanical interlocking leads to high interfacial shear stress. The MD simulation also showed that the mismatch of the coefficients of thermal expansions between $\mathrm{CNT}$ and polymer matrix also promotes the stress transfer ability. Xu and Buehler [10] have studied the thermal resistance of the two interacting CNTs, with and without polymer, which shows that the thermal resistance of the CNT junctions can be significantly improved through altering the number of polymer molecules. Recently, Zhang et al. [13] have studied the effects of the dispersion of polymer-wrapped two neighbouring SWNTs on nanoengineering load transfer. They found that dispersion angle of the polymer wrapped SWNTs dominates the load transfer. Besides, the self-repairing function of the system is found due to the nonbond interaction between the polymer and the SWNTs.

Frankland et al. [7] modeled a single armchair CNT by $\mathrm{MD}$ and found that the shear strength increases 10 times just by forming less than $1 \%$ cross-links between the CNT and the polymer. Through the MD simulation, Chowdhury and Okabe [47] also investigated the influence of the chemical cross-links at the interface and found that forming a few cross-links between the CNT and the polymer, the shear strength increases from $310 \mathrm{MPa}$ to $1630 \mathrm{MPa}$. Liu et al. [11] obtained the shear strength by pullout test of SWNT from covalent bonded polymer with an MD simulation. They found that the increased interface shear strength can either enhance or reduce the damping stability of the composite. Other researchers like Kuang, Tsai, and Odegard adopted MD methods to study the properties of the nanocomposites and interface between the CNT and the polymer [48-50].

However, the large number of particles and the short time steps involved in MD simulations call for powerful computational facilities, which are typically provided through parallel computing. The simulation time may be shortened by employing typical modeling strategies, such as adopting periodic boundary conditions [51] and reducing the cutoff distance assumption [52]. A more detailed discussion can be found in the Table 1.

3.1.2. Coarse Grain Simulation. A coarse grain (CG) approach is used to develop the tools for investigating the material properties. It is also used to study the underlying mechanical behavior typically required for material design that systematically integrates characteristic chemical responses. The CG models provide efficient means to simulate and investigate systems in which the desired behavior, property, or response is inherently at the mesoscale-those that are both inaccessible to full atomistic representations and inapplicable to continuum theory. The CG techniques are developed to map atomistic systems onto systems with coarser resolution. Therefore, a developed CG model can only reflect the behavior included in their governing potentials and associated parameters, and consequently, the source of such parameters typically determines the accuracy and utility of the CG model $[53,54]$. It is emphasized that not all systems will benefit from a coarse-grain representation, and prudent consideration must be given regarding the system characterization and the intent of the simulations. Typical motivations for a CG approach include the following:

(1) inaccessible time scale and length scale for phenomenon or behavior via full atomistic representation;

(2) focus on bulk properties and/or mechanical behavior rather than on molecular structure and/or chemical interactions;

(3) desire for a direct simplified analysis of simulation results and system behavior.

In the past decade, various CG molecular dynamics methods were extensively used to study the thermomechanical properties of polymers, proteins, lipids, and biomaterials [53-56].

Müller-Plathe's group reported many interesting properties of different polymers (e.g., thermal conductivity, chain stiffness) by the CG MD simulations [57-59], in which the CG potentials were developed and their parameters were provided based on the reverse-mapping procedure by validating from full-atom MD simulations. Kremer's group developed two kinds of CG potentials for polystyrene, and the thermodynamical and the chemical properties were studied by CG MD simulations $[60,61]$. Theodoru and Fermeglia investigated the multiscale modeling for polymer systems using CG $\mathrm{MD}$ and full-atom MD methods as well as Monte Carlo (MC) method [62, 63]. Nielsen et al. [64] calculated surface tension in bulk PE by CG MD simulations, which considerably reduces the amount of numerical computing required in the MD simulations. Recently, Zhao et al. [65] have developed a more accurate CG potential to study the thermomechanical properties (such as bulk density, glass transition temperature, expansion coefficient, Young's moduli, and yield stress) of bulk polyethylene (PE). The size-dependent mechanical properties of compressive stress-strain curves for PE particles have also been obtained by the CG MD method [66].

As it can be seen, all aforementioned studies are performed on pure polymer. The main reason for this is that the first step in studying interaction between CNT and surrounding polymer using CG MD is constructing a reasonable model for the chain of polymer. Therefore, numerous studies have been conducted on pure polymer.

Buehler's group developed the various CG MD potentials to characterize the nanoindentation of single- and doublecarbon nanotubes (CNTs) buckypaper [67]. Based on the CG model, the mechanical properties of CNT networks were obtained, and the microstructural evolution and failure mechanism were discussed [68]. Afterwards, the viscoelasticity of the CNTs networks was reported by the CG MD simulations [69].

However, all the previous CG models are used in polymers and CNTs, respectively, while the CNT reinforced polymer composites are scarcely studied by CG MD simulations since the Lennard-Jones (LJ) potentials between CG beads of polymers and CNTs are not obtained yet. Nevertheless, 
TABLE 1: Conducted molecular dynamics modeling of interaction between the CNT and the polymer.

\begin{tabular}{|c|c|c|c|c|c|c|c|}
\hline Researcher(s) & Year & Reinforcement & Matrix & $\begin{array}{l}\text { Simulation } \\
\text { size }\end{array}$ & Simulation setup & Conclusion & $\begin{array}{c}\text { Shortcomings/ } \\
\text { advantages }\end{array}$ \\
\hline Liao and $\mathrm{Li}$ [6] & 2001 & $\begin{array}{l}\text { SWNT and } \\
\text { DWNT }\end{array}$ & $\begin{array}{l}\text { One chain of } 2 \text { to } \\
80 \text { mer polystyrene }\end{array}$ & $\begin{array}{c}20 \AA \text { long } \\
\text { CNT }\end{array}$ & $\begin{array}{l}\text { Pullout without } \\
\text { cross-link between } \\
\text { CNTs and matrix }\end{array}$ & $\begin{array}{c}\text { Full-atom and } \\
\text { nonbond interaction. } \\
\text { Pullout shear } \\
\text { strength of } 160 \mathrm{MPa}\end{array}$ & $\begin{array}{l}\text { More accurate while } \\
\text { limited to small size }\end{array}$ \\
\hline $\begin{array}{l}\text { Frankland et } \\
\text { al. [7] }\end{array}$ & 2002 & $\begin{array}{l}53 \AA \text { A long (10, } \\
\text { 10) SWNT }\end{array}$ & $\begin{array}{l}178 \text { chains of } 42 \text { mer } \\
\text { polyethylene }\end{array}$ & $\begin{array}{l}53 \AA \text { long } \\
\text { SWNT }\end{array}$ & $\begin{array}{l}\text { Pullout for both } \\
\text { crystalline and } \\
\text { amorphous } \\
\text { polymer }\end{array}$ & $\begin{array}{c}\text { An order of } \\
\text { magnitude increase } \\
\text { in shear strength } \\
\text { with less than } 1 \% \\
\text { cross-link between } \\
\text { SWNT polymer } \\
\end{array}$ & $\begin{array}{l}\text { Close to the actual } \\
\text { situation, limited to } \\
\text { small scale }\end{array}$ \\
\hline Wong et al. [8] & 2003 & SWNT & Epoxy, polystyrene & $\begin{array}{l}31 \AA ̊ \text { long } \\
\text { CNT }\end{array}$ & $\begin{array}{l}\text { Ununiformed } \\
\text { SWNT with two } \\
\text { different diameters. }\end{array}$ & $\begin{array}{l}\text { Local nonuniformity } \\
\text { of CNT and } \\
\text { mismatch of the } \\
\text { coefficients of } \\
\text { thermal expansions } \\
\text { lead to high } \\
\text { interfacial shear } \\
\text { stress }\end{array}$ & $\begin{array}{l}\text { New aspect for } \\
\text { analyzing the } \\
\text { enhanced effect }\end{array}$ \\
\hline Chen et al. [9] & 2008 & $\begin{array}{c}49.19 \AA \text { A long } \\
(10,10) \text { SWNT }\end{array}$ & $\begin{array}{l}\text { One chain of } 10 \text { mer } \\
\text { polyphenylacetylene }\end{array}$ & $\begin{array}{c}49.19 \AA \mathrm{CNT} \\
\text { with Single } \\
\text { polymer } \\
\text { chain }\end{array}$ & $\begin{array}{l}\text { SWNTs with } \\
\text { different chirality }\end{array}$ & $\begin{array}{c}\text { Strongest adhesion } \\
\text { for armchair-type } \\
\text { SWNT }\end{array}$ & $\begin{array}{l}\text { Clear analysis but } \\
\text { the size is too small }\end{array}$ \\
\hline $\begin{array}{l}\text { Xu and } \\
\text { Buehler [10] }\end{array}$ & 2009 & $(10,10)$ SWNT & $\begin{array}{l}\text { Polyethylene, } \\
\text { various chain } \\
\text { lengths }\end{array}$ & $\begin{array}{l}25 \text { to } 75 \mathrm{~nm} \\
\text { long CNT }\end{array}$ & $\begin{array}{c}\text { Overlapped } \\
\text { SWNTs with or } \\
\text { without polymers }\end{array}$ & $\begin{array}{l}\text { The thermal } \\
\text { resistance of CNT } \\
\text { junctions can be } \\
\text { significantly } \\
\text { improved through } \\
\text { modifying the } \\
\text { molecular structure } \\
\text { at the interface }\end{array}$ & $\begin{array}{l}\text { New idea with } \\
\text { full-atom analysis, } \\
\text { heavy calculations }\end{array}$ \\
\hline Liu et al. [11] & 2011 & $(10,10)$ SWNT & $\begin{array}{l}\text { EPON } 862 \text { and } \\
\text { EPI-CURE curing } \\
\text { agent }\end{array}$ & $\begin{array}{c}4.6 \mathrm{~nm} \times \\
4.6 \mathrm{~nm} \times \\
6.3 \mathrm{~nm}\end{array}$ & $\begin{array}{l}\text { SWNT pullout test } \\
\text { is preformed by } \\
\text { MD method, then } \\
\text { the shear strength } \\
\text { is applied to a } \\
\text { micromechanical } \\
\text { damping model }\end{array}$ & $\begin{array}{l}\text { The increased } \\
\text { interface shear } \\
\text { strength can either } \\
\text { enhance or reduce } \\
\text { the damping stability } \\
\text { of the composite }\end{array}$ & $\begin{array}{l}\text { Useful method to } \\
\text { determine the } \\
\text { parameters for } \\
\text { multiscale } \\
\text { hierarchical model }\end{array}$ \\
\hline $\begin{array}{l}\text { Rahmat and } \\
\text { Hubert [12] }\end{array}$ & 2012 & $\begin{array}{c}52.69 \AA(10,0) \\
\text { SWNT }\end{array}$ & $\begin{array}{c}9 \text { Chains of } 32 \text { mer } \\
\text { PMMA }\end{array}$ & Varied & $\begin{array}{l}\text { Introduction and } \\
\text { pullout test with } \\
\text { optimized } \\
\text { simulation phases }\end{array}$ & $\begin{array}{c}\text { Interfacial binding } \\
\text { energy of } \\
0.39 \mathrm{kcal} / \mathrm{mol} \AA^{2} \text { for } \\
\text { no covalent } \\
\text { interaction } \\
\end{array}$ & $\begin{array}{l}\text { Common MD } \\
\text { simulation. }\end{array}$ \\
\hline $\begin{array}{l}\text { Zhang et al. } \\
\text { [13] }\end{array}$ & 2013 & $\begin{array}{c}59.03 \AA \text { long } \\
\text { armchair }(5,5)\end{array}$ & $\begin{array}{l}\text { Chain length of } 20 \\
\quad 40,60\end{array}$ & $\begin{array}{l}59.03 \text { Å long } \\
\text { SWNT }\end{array}$ & $\begin{array}{c}\text { Varying the } \\
\text { dispersion angle of } \\
\text { two neighbouring } \\
\text { SWNTs }\end{array}$ & $\begin{array}{l}\text { Dispersion angle of } \\
\text { polymer wrapped } \\
\text { SWNTs dominates } \\
\text { the load transfer }\end{array}$ & $\begin{array}{l}\text { United atom (UA) } \\
\text { method, can apply } \\
\text { to large scale }\end{array}$ \\
\hline
\end{tabular}

this issue will likely be addressed soon, since studies in this direction show high potential and important applications.

Because the CG models normally lose more detailed information among the beads (such as dihedral and improper information), accurate quantitative nonlinear mechanical properties of materials cannot be accurately given in the CG MD simulations.
3.1.3. Density Functional Theory. Density functional theory (DFT) studies the electronic structure of atomic scale systems based on quantum mechanics. The DFT models commonly use approximations, such as local density approximation (LDA), in which only the value of the electronic density at each point in the space is considered compared to the MD simulation. 
$\mathrm{Ab}$ initio simulations use DFT and are able to converge to the accurate solution. However, these simulations are computationally expensive and grow in size with the power 3-7 of the number of basic functions. Therefore, the ab initio simulations are fairly small in size and use various techniques to reduce the computational costs. For example, during some stages of the simulation, only some part of the system may be optimized, or atomic relaxation may not be allowed and molecules may be considered to be rigid.

$\mathrm{Ab}$ initio models have studied various types of nanotubepolymer interaction including van der Waals and chemical functionalization. The results of these models are typically presented in the form of local density of state for the nanotube and the nanocomposite, band structures, charge density contours, and binding energy as a function of distance [70,71]. In the work of Kim et al. [70], the encapsulation energy of a single $\left(\mathrm{C}_{2} \mathrm{H}_{2}\right)$ molecule into the nanotube and the formation energy of $\left(\mathrm{C}_{2} \mathrm{H}_{2}\right) @ \mathrm{CNT}$ are calculated. In the work of Mylvaganam and Zhang [71], the possible ways of functionalizing CNTs are investigated with the aid of the density functional theory. Using the methoxy radical and the secondary butyl anion as initiators and their reactions with ethylene and epoxide, this leads to polyethylene- and polyepoxide-grafted nanotubes.

Most of DFT models are based on static or zero Kelvin structures and are still limited to small systems. Hence, when using DFT models, the strength of these models (accurate prediction of atomic structure) along with the inevitably expensive computations should be considered.

3.1.4. The Challenges of Atomistic Modeling. As it has been stated, atomic modeling has the accuracy and high flexibility to observe effects of various parameters on the mechanical properties of the nanocomposites and the interphase between the CNT and the polymer. But a lot of limiting factors are affecting these methods. For example, most of the published papers that have used these methods are using SWCNTs with a length less than $10 \mathrm{~nm}$. In addition, the number of polymer chains considered is less than 100 .

This is because atomistic modeling methods are not able to handle a large number of atoms due to increasing computational costs. Also the formulas used in these methods often have high complexity. Due to these limitations, continuum models have been used to overcome the aforementioned difficulties.

3.2. Continuum Modeling. Continuum modeling of CNT and surrounding polymer interactions is accomplished either analytically or numerically.

3.2.1. Analytical Modeling. In studies by analytical methods to model interphase between the carbon nanotube and the polymer, most of the researchers have tried to use the existing microscale rules in the field composites. These rules known as micromechanics rules cannot be directly applied to the nanoscale due to assumptions which are no longer valid. These involve the uniform distribution of fibers in polymer and perfect bonding between CNT and polymer, as well as considering reinforcement phase as a continuum medium which is applicable to fibers. In other words, direct use of micromechanics equations for CNTRP will simply neglect the lattice structure of CNT and consider CNT as a solid fiber. Moreover, micromechanics equations assume perfect bonding between the reinforcing agents and the surrounding polymer. This is not pertinent to the interphase between the CNT and the surrounding polymer, which interact through nonbonded van der Waals (vdW) forces.

Some researchers have proposed that a material region between CNT and surrounding polymer should be considered, but the way to treat the properties of this specific material region remains to be determined. The associated properties are selected between matrix and CNT Young's modulus without any experimental basis. Actually, at the scale of nano, there is no material region for interphase between CNT and polymer, but instead, the region is a molecular interaction space. Some others suggested some corrective factors in order to reflect the imperfection bonding between CNT and polymer, but these factors are also obtained on the basis of curvefitting data.

Actually, CNTs interact with the surrounding polymer through vdW forces. This will cause the CNT to slide in the polymer matrix when subjected to axial loading. Therefore, the assumption of perfect bonding between CNT and polymer is not valid. Thus, some researchers try to incorporate vdW interactions between CNT and polymer in their modeling methods. Cohesive zone models have been widely used in the continuum study of interface debonding and sliding in composites. A cohesive zone model assumes a relation between the normal (and shear) traction(s) and the opening (and sliding) displacement(s). When implemented in the finite element method, the cohesive zone model is capable of simulating interface debonding and sliding. The existing cohesive models are all phenomenological, because it is difficult to obtain directly the cohesive laws for interfaces. Jiang et al. [14] have developed the cohesive zone model for the interface between the CNT and the polymer. They have calculated the total energy of all the atoms by integrating over the volume and by differentiation from the movement of the plates in the vertical and in the tangential direction. Finally, they report the relation between the normal (and shear) traction(s) and the opening (and sliding) displacement(s) across the interface in terms of the area density of CNT and the volume density of polymer, as well as the parameters in the vdW force.

Assuming an interface which contains vdW interactions, Tan et al. [15] have attempted to calculate the relationship between the stress and the strain in nanocomposites. In this regard, the rules set by Jiang have helped to describe the relationship between the stress and the strain at the interface.

Seidel and Lagoudas [16] tried to evaluate the effect of an interphase on the mechanical properties of nanocomposites. They have considered interphase as a physical environment and assumed elastic modulus of this area 0.1 to 10 times the elastic modulus of the matrix. Then, the effects of interphase properties on nanocomposites were examined using a concentric cylinder model.

The Shear-Lag model was also employed by some other researchers to study the interaction between CNT and 
TABLE 2: Conducted analytical modeling of interaction between CNT and polymer.

\begin{tabular}{|c|c|c|c|c|c|c|}
\hline Researcher(s) & Year & Interface or interphase & Type of interaction & Thickness & $\begin{array}{l}\text { Interphase } \\
\text { modulus }\end{array}$ & $\begin{array}{l}\text { Method of } \\
\text { modeling }\end{array}$ \\
\hline Jiang et al. [14] & 2006 & Interface & $\mathrm{vdW}$ & N/A & N/A & Cohesive energy \\
\hline Tan et al. [15] & 2007 & Interface & vdW & N/A & $\begin{array}{l}\text { Imperfection } \\
\text { coefficient }\end{array}$ & Elasticity \\
\hline $\begin{array}{l}\text { Seidel and } \\
\text { Lagoudas [16] }\end{array}$ & 2006 & Interphase & Perfect & $0.5 \times R_{\mathrm{NT}}$ to $4 \times R_{\mathrm{NT}}$ & $0.1 \times E_{m}$ to $10 \times E_{m}$ & $\begin{array}{c}\text { Composite } \\
\text { cylinders } \\
\text { micromechanics }\end{array}$ \\
\hline Tsai and Lu [17] & 2009 & Interphase & Perfect & $0.01 \mathrm{~nm}$ & $\begin{array}{c}G_{\text {int }}=0.13,0.286 \\
4.2,370 \mathrm{GPa}\end{array}$ & $\begin{array}{c}\text { Elasticity (shear } \\
\text { lag) }\end{array}$ \\
\hline Nairn [18] & 2011 & Interface or interphase & Imperfect & N/A & $\begin{array}{c}\text { Imperfection } \\
\text { coefficient }\end{array}$ & $\begin{array}{l}\text { Shear lag } \\
\text { (elasticity) }\end{array}$ \\
\hline $\begin{array}{l}\text { Shokrieh and } \\
\text { Mahdavi [19] }\end{array}$ & 2011 & Interphase & $\mathrm{vdW}$ & $0.17 \mathrm{~nm}$ & $\begin{array}{l}\text { Function of } l_{\mathrm{NT}} \\
\quad \text { and } R_{\mathrm{NT}}\end{array}$ & $\begin{array}{l}\text { Equivalent fiber } \\
\quad \text { (elasticity) }\end{array}$ \\
\hline $\begin{array}{l}\text { Barai and Weng } \\
{[20]}\end{array}$ & 2011 & Interface & Perfect & $\mathrm{N} / \mathrm{A}$ & $\begin{array}{l}\text { Imperfection } \\
\text { coefficient }\end{array}$ & Elasticity \\
\hline
\end{tabular}

polymer $[17,18]$. This model is usually used for long fiber composites at microscale, and it is not suitable for CNT reinforced composites wherein the effective scale is nano. This model has been basically developed for continuum reinforcing agent, and it could not be applied to the lattice structure of CNT. Tsai and Lu [17] took into account the interphase as a physical environment. They used different values for the shear modulus of interphase and investigated the effect of interphase on load transfer efficiency in the CNTs reinforced nanocomposites using the conventional shear lag model. Nairn [18] considered the effect of the interphase by a coefficient varying between zero and infinity in the formula for calculating the modulus of the nanocomposite.

Shokrieh and Mahdavi [19] considered a virtual material region for the interphase. They have obtained the properties of an equivalent fiber using the elasticity approach when the interactions are assumed to be vdW. The obtained results were in a very good agreement with the developed multiscale FE modeling by Shokrieh and Rafiee [29].

Barai and Weng [20] studied the mechanical properties of carbon nanotube with elasticity equations. They fit an imperfect factor to their equations and investigated the effect of the interphase by changing this ratio. It was reported that when the imperfection at the interface increases, the effective Young's modulus of the composite decreases and those CNTs with very weak interfaces will behave like voids. It should be pointed out that since Barai and Weng [20] have considered perfect bonding between CNT and polymer, they are addressing higher stress transferability from matrix to CNT in contrast to what has been reported by Tan et al. [15] through weak vdW interactions.

An overview of conducted analytical modeling to study the interaction between the CNT and the surrounding polymer is summarized in Table 2.

As it can be understood from the literature survey, different values have been considered for the modulus and also for the thickness of interphase. In some investigations, the influence of the interphase region has just been simulated using some correction factor to reflect the imperfection.
3.2.2. Numerical Modeling. Several researchers have used numerical modeling employing the finite element analysis to study the interaction between the CNT and the surrounding polymer. Some investigators considered an intermediate region between the CNT and the polymer as a separate phase and used various elements like link, truss, rod, beam, and spring with different properties describing the molecular interaction between the CNT atoms and the surrounding polymer chains. Some others have ignored this region in the modeling and simply assumed a perfect bonding between nanotube and the polymer $[21,22]$. However, since it is well known that perfect bonding between the nanotubes and the resin is not a valid assumption, these studies will not be reviewed in this report.

Haque and Ramasetty [21] obtained shear and axial stresses at the interface without considering interphase using the equilibrium and the elastic equations.

Wan and his coworkers [27] calculated the properties of the RVE of CNTRP considering interphase with the thickness of $1.357 \mathrm{~nm}$. The modulus of interphase has been varied between 0.3 and 10 times the elastic modulus of the matrix. They considered the interphase as an isotropic material with constant properties.

$\mathrm{Li}$ and Chou [23] consider the vdW interactions between the nanotube and the inner surface of the surrounding polymeric matrix and used nonlinear truss elements to simulate $\mathrm{vdW}$ interactions. They considered the thickness of this region as $0.17 \mathrm{~nm}$ and achieved the properties of the elements using Lennard-Jones potential.

Pourakbar and his coworkers [24] have studied the effect of functionalized nanotube on the properties of a RVE. They modeled covalent bonds between the nanotubes and the resin with beam elements and used properties that were derived from molecular mechanics approach. They found out that by increasing the population of covalent bonds at the interphase, the stress transferability from matrix to CNT is increased and it will converge to the results achieved from the rule of mixture, even though the rule of mixture is not valid at nanoscale. 
TABLE 3: Finite element modeling of CNT and polymer interaction.

\begin{tabular}{|c|c|c|c|c|c|c|}
\hline Researcher(s) & Year & $\begin{array}{l}\text { Interface or } \\
\text { interphase }\end{array}$ & Type of interaction & Thickness & $E_{\text {int }}$ and $G_{\text {int }}$ & Element \\
\hline Haque and Ramasetty [21] & 2005 & Interface & Perfect & N/A & $\mathrm{N} / \mathrm{A}$ & N/A \\
\hline Wan et al. [27] & 2005 & Interphase & Perfect & $1.357 \mathrm{~nm}$ & $10 \times E_{m}$ and $0.3 \times E_{m}$ & N/A \\
\hline Li and Chou [23] & 2006 & Interphase & vdW & $0.17 \mathrm{~nm}$ & Derived from LJ potential & Truss rod \\
\hline Pourakbar et al. [24] & 2008 & Interphase & Functionalized & $1.522 \mathrm{~nm}$ & From $\mathrm{MD}$ & Beam \\
\hline Giannopoulos et al. [25] & 2009 & Interphase & vdW & $0.17 \mathrm{~nm}$ & Parametric & Joint element \\
\hline Montazeri and Naghdabadi [26] & 2009 & Interphase & $\mathrm{vdW}$ & $0.34 \mathrm{~nm}$ & Derived from LJ potential & Spring \\
\hline Kulkarni et al. [22] & 2010 & Interface & Perfect & 0 & Different value & Interface element \\
\hline Needleman et al. [28] & 2010 & $\begin{array}{c}\text { Interphase } \\
\text { and interface }\end{array}$ & Perfect & Variable & Linearly between $E_{m}$ and $E_{n}$ & N/A \\
\hline Shokrieh and Rafiee [29] & 2010 & Interphase & vdW & $0.17 \mathrm{~nm}$ & Derived from LJ potential & Nonlinear spring \\
\hline Ayatollahi et al. [30] & 2011 & Interphase & Perfect & $0.15 \mathrm{~nm}$ & $1,5,10$ and $15 \mathrm{GPa}$ & Solid \\
\hline Wernik and Meguid [31] & 2011 & Interphase & vdW & $0.38 \mathrm{~nm}$ & Derived from LJ potential & Truss rod \\
\hline
\end{tabular}

Giannopoulos et al. [25] considered the modulus of interphase as a function of the matrix and CNT's modulus. In their work, joint elements were used to simulate the interphase between the CNT and the matrix in radial direction along the nanotube. The results reveal a high dependence of the longitudinal modulus of the nanocomposites on the stiffness of the interfacial region. The high stiffness values of the joint elements (perfect bonding between the CNT and matrix) lead to the similar results obtained by the rule of mixture. In contrast, when the nanotube is very softly bonded to the matrix, no substantial enhancement in mechanical properties is observed.

Montazeri and Naghdabadi [26] have used one-dimensional spring elements to model the interphase. They have achieved the associated properties of the spring elements from Lennard-Jones potential.

Kulkarni et al. [22] have studied the properties of the RVE by considering the interface and the force region between the nanotubes and the resin. They have used interface elements in the modeling and used varied strength and investigated the effect of that on the properties of RVE. They reported that the modulus of the nanocomposite increases with increasing interface strength. The rate of increase in the modulus of the nanocomposite becomes smaller, as the interface strength increases.

Needleman and his coworkers [28] calculated the properties of RVEs. They have simulated the interphase with a variable thickness and nonlinear properties between nanotubes and the resin. They considered a perfect bonding between the nanotubes and the resin and investigated the influence of interphase strength on the properties of investigated RVE.

Shokrieh and Rafiee [29] proposed a model for interphase where bonds between nanotubes and the resin would be updated when the applied strain evolves. Consequently, the model is called Adaptive vdW Interaction (AVI). They have used nonlinear spring elements capturing vdW interactions. In other words, when the applied strain is increased, the instantaneous status of each vdW bond is updated in accordance with the governing equation of the vdW interaction. They have observed that the rule of mixture will overestimate the results. This stems from this fact that the rule of mixture considers perfect bonding between CNT and polymer. The results demonstrate the importance of considering vdW based interphase.

Wernik and Meguid [31] offered the same model as Shokrieh and Rafiee's model for interphase. The only difference was that they used truss elements instead of spring elements for interphase. They reported the same trend reported by Shokrieh and Rafiee in comparison with the rule of mixture.

Ayatollahi et al. [30] obtained properties of RVE considering interphase with 3 times the thickness of the carbon nanotube and different elastic moduli as 0.2 , 2, and $20 \mathrm{GPa}$. They investigated the effect of the interphase on the properties of the RVE with different values of elasticity modulus of the interphase. They found out that generally, by increasing the modulus of interphase, the load transfer between CNT and polymer will be improved and the modulus of nanocomposites will be increased.

An overview of the finite element modeling studies on the interphase region between the CNT and the polymer is presented in Table 3.

It can be seen from Table 3 that those who have assumed the interactions between CNT and surrounding polymer take place through force environment also used interface to simulate the interaction between CNT and polymer. They have observed that increasing the interface strength leads to convergence of the modulus of nanocomposite to that obtained by the rule of mixtures, since they are addressing perfect bonding between CNT and polymer.

On the other side, when the interaction between CNT and surrounding polymer is treated as a physical environment, an interphase between CNT and surrounding polymer is modeled. The modeling of interphase appears either in the form of intermediate material region between CNT and polymer or in the form of connection links between CNT and polymer capturing by spring, truss, or beam elements. The former is called continuum modeling of interphase, while the latter is called semicontinuum modeling of the interphase region. 


\section{Conclusion}

The modeling of interface/interphase region between the CNT and the polymer is reviewed in this scientific review. The theoretical studies are divided into two main groups: atomistic modeling and continuum modeling.

Atomistic modeling can well capture the shear strength and bonding energy of the interface between the CNT and the surrounding polymer matrix in nanoscale. Moreover, the obtained properties can be upscaled to the macroscale model. The united atom (UA) and coarse grain (CG) methods are promising solutions to extend the simulation to microscale. However, the beads-incorporated simulation still requires a huge amount of calculation and is limited to small length scale compared to the continuum model. Moreover, obtaining the accurate nonlinear mechanical properties is one of the limitations of using the CG method. As a consequence, continuum modeling is used by different researchers to overcome mentioned difficulties. But the degree to which the continuum modeling can truly predict the actual behavior of CNT at nanoscale has to be carefully studied.

Continuum modeling can be done either analytically or numerically. The analytical modeling of the continuum model usually considers simply perfect bonding between the $\mathrm{CNT}$ and the surrounding polymer and tries to capture the influence of the imperfect bonding between the CNT and the polymer by introducing some correction factor in the micromechanics rules.

The numerical continuum modeling which takes place using FEM is divided into three main groups. The first group takes into account the force interaction between CNT and polymer through interface without considering any distinct material region. In this group, the researchers usually replace CNT with a continuum medium for sake of simplicity, and thus their results approach the values reported by rule of mixture. It is worth mentioning that the basic assumptions of the rule of mixture are not valid for the case of CNT. Micromechanics consider continuum medium for the reinforcing agent, which is not pertinent to the case of CNT, and also it assumes perfect bonding between CNT and surrounding polymer when instead CNT naturally interacts with the polymer through weak vdW interactions.

The second group considers an intermediate material region between the $\mathrm{CNT}$ and the polymer as a continuum medium and uses different values for the properties of this distinct region. Both the thickness and the mechanical properties of this region are under question in this category of study, as the selection has no experimental basis. Almost all studies selected Young's modulus of the interphase region as a varying parameter between Young's modulus of resin and CNT.

The first two groups can be also found in the field of analytical modeling as well.

The third group uses different elements consisting of joint, bar, truss, spring, and beam to construct the interaction between the CNT and the surrounding polymer. The properties of these elements are obtained from molecular space, and therefore equivalent structural members are employed. Due to the specific fact that a continuum region has not been considered for the interphase region and actually continuum elements are used for the purpose of molecular interaction, this group of study is also called as semicontinuum modeling. Instead of considering a continuum material region, just vdW interactions or covalent bonds between CNT and surrounding polymer are simulated using continuum elements. The former is the natural interaction between CNT and polymer in the absence of chemical functionalization, while the latter captures functionalized CNT. Semicontinuum modeling has received more interest among researchers in the recent years.

It can be concluded that despite the fact that atomistic modeling can provide an accurate result on local properties and interactions between CNT and polymer, it is computationally intensive, and it is limited to very short time and small length scales. However, continuum modeling can be considered as a reasonable compromise in modeling, while it is focusing on global behavior rather than local one. Among developed continuum modeling, the more preferred approach is semicontinuum modeling, wherein CNT is treated as a lattice structure, and the interactions between $\mathrm{CNT}$ and surrounding polymer are replaced by continuum elements.

\section{References}

[1] J. Gou, B. Minaie, B. Wang, Z. Liang, and C. Zhang, "Computational and experimental study of interfacial bonding of single-walled nanotube reinforced composites," Computational Materials Science, vol. 31, no. 3-4, pp. 225-236, 2004.

[2] E. T. Thostenson and T. Chou, "Aligned multi-walled carbon nanotube-reinforced composites: processing and mechanical characterization," Journal of Physics D, vol. 35, no. 16, pp. L77L80, 2002.

[3] C. Bower, R. Rosen, L. Jin, J. Han, and O. Zhou, "Deformation of carbon nanotubes in nanotube-polymer composites," Applied Physics Letters, vol. 74, no. 22, pp. 3317-3319, 1999.

[4] C. A. Cooper, S. R. Cohen, A. H. Barber, and H. D. Wagner, "Detachment of nanotubes from a polymer matrix," Applied Physics Letters, vol. 81, no. 20, pp. 3873-3875, 2002.

[5] D. Qian and E. C. Dickey, "In-situ transmission electron microscopy studies of polymer-carbon nanotube composite deformation," Journal of Microscopy, vol. 204, no. 1, pp. 39-45, 2001.

[6] K. Liao and S. Li, "Interfacial characteristics of a carbon nanotube-polystyrene composite system," Applied Physics Letters, vol. 79, no. 25, pp. 4225-4227, 2001.

[7] S. J. V. Frankland, A. Caglar, D. W. Brenner, and M. Griebel, "Molecular simulation of the influence of chemical cross-links on the shear strength of carbon nanotube-polymer interfaces," Journal of Physical Chemistry B, vol. 106, no. 12, pp. 3046-3048, 2002.

[8] M. Wong, M. Paramsothy, X. J. Xu, Y. Ren, S. Li, and K. Liao, "Physical interactions at carbon nanotube-polymer interface," Polymer, vol. 44, no. 25, pp. 7757-7764, 2003.

[9] H. Chen, Q. Xue, Q. Zheng, J. Xie, and K. Yan, "Influence of nanotube chirality, temperature, and chemical modification on the interfacial bonding between carbon nanotubes and polyphenylacetylene," Journal of Physical Chemistry C, vol. 112, no. 42, pp. 16514-16520, 2008. 
[10] Z. Xu and M. J. Buehler, "Nanoengineering heat transfer performance at carbon nanotube interfaces," ACS Nano, vol. 3, no. 9, pp. 2767-2775, 2009.

[11] A. Liu, K. W. Wang, and C. E. Bakis, "Effect of functionalization of single-wall carbon nanotubes (SWNTs) on the damping characteristics of SWNT-based epoxy composites via multiscale analysis," Composites A, vol. 42, no. 11, pp. 1748-1755, 2011.

[12] M. Rahmat and P. Hubert, "Molecular dynamics simulation of single-walled carbon nanotube-PMMA Interaction," Journal of Nano Research, vol. 18-19, pp. 117-128, 2012.

[13] Y. Zhang, J. Zhao, N. Wei, J. Jiang, Y. Gong, and T. Rabczuk, "Effects of the dispersion of polymer wrapped two neighbouring single walled carbon nanotubes (SWNTs) on nanoengineering load transfer," Composites B, vol. 45, no. 1, pp. 1714-1721, 2013.

[14] L. Y. Jiang, Y. Huang, H. Jiang et al., "A cohesive law for carbon nanotube/polymer interfaces based on the van der Waals force," Journal of the Mechanics and Physics of Solids, vol. 54, no. 11, pp. 2436-2452, 2006.

[15] H. Tan, L. Y. Jiang, Y. Huang, B. Liu, and K. C. Hwang, "The effect of van der Waals-based interface cohesive law on carbon nanotube-reinforced composite materials," Composites Science and Technology, vol. 67, no. 14, pp. 2941-2946, 2007.

[16] G. D. Seidel and D. C. Lagoudas, "Micromechanical analysis of the effective elastic properties of carbon nanotube reinforced composites," Mechanics of Materials, vol. 38, no. 8-10, pp. 884907, 2006.

[17] J.-L. Tsai and T.-C. Lu, "Investigating the load transfer efficiency in carbon nanotubes reinforced nanocomposites," Composite Structures, vol. 90, no. 2, pp. 172-179, 2009.

[18] J. A. Nairn, "Aspect ratio requirements for nanotube-reinforced, polymer-matrix composites," Composites $A$, vol. 42, no. 11, pp. 1850-1855, 2011.

[19] M. M. Shokrieh and S. M. Mahdavi, "Micromechanical model to evaluate the effects of dimensions and interphase region on the elastic modulus of CNT/polymer composites," Modarres Mechanical Engineering, vol. 3, pp. 13-25, 2011.

[20] P. Barai and G. J. Weng, "A theory of plasticity for carbon nanotube reinforced composites," International Journal of Plasticity, vol. 27, no. 4, pp. 539-559, 2011.

[21] A. Haque and A. Ramasetty, "Theoretical study of stress transfer in carbon nanotube reinforced polymer matrix composites," Composite Structures, vol. 71, no. 1, pp. 68-77, 2005.

[22] M. Kulkarni, D. Carnahan, K. Kulkarni, D. Qian, and J. L. Abot, "Elastic response of a carbon nanotube fiber reinforced polymeric composite: a numerical and experimental study," Composites B, vol. 41, no. 5, pp. 414-421, 2010.

[23] C. Li and T. Chou, "Multiscale modeling of compressive behavior of carbon nanotube/polymer composites," Composites Science and Technology, vol. 66, no. 14, pp. 2409-2414, 2006.

[24] K. Pourakbar, N. JamilPour, A. R. Najafi, G. Rouhi, A. Arshi, and A. Fereidoon, "A finite element model for estimating Young's modulus of carbon nanotube reinforced composites incorporating elastic cross-links," International Journal of Mechanical, Industrial and Aerospace Engineering, pp. 172-175, 2008.

[25] G. I. Giannopoulos, S. K. Georgantzinos, and N. K. Anifantis, "A semi-continuum finite element approach to evaluate the Young's modulus of single-walled carbon nanotube reinforced composites," Composites B, vol. 41, no. 8, pp. 594-601, 2010.

[26] A. Montazeri and R. Naghdabadi, "Study the effect of viscoelastic matrix model on the stability of CNT/polymer composites by multiscale modeling," Polymer Composites, vol. 30, no. 11, pp. 1545-1551, 2009.

[27] H. Wan, F. Delale, and L. Shen, "Effect of CNT length and CNT-matrix interphase in carbon nanotube (CNT) reinforced composites," Mechanics Research Communications, vol. 32, no. 5, pp. 481-489, 2005.

[28] A. Needleman, T. L. Borders, L. C. Brinson, V. M. Flores, and L. S. Schadler, "Effect of an interphase region on debonding of a CNT reinforced polymer composite," Composites Science and Technology, vol. 70, no. 15, pp. 2207-2215, 2010.

[29] M. M. Shokrieh and R. Rafiee, "Prediction of mechanical properties of an embedded carbon nanotube in polymer matrix based on developing an equivalent long fiber," Mechanics Research Communications, vol. 37, no. 2, pp. 235-240, 2010.

[30] M. Ayatollahi, S. Shadlou, and M. Shokrieh, "Multiscale modeling for mechanical properties of carbon nanotube reinforced nanocomposites subjected to different types of loading," Composite Structures, vol. 93, no. 9, pp. 2250-2259, 2011.

[31] J. M. Wernik and S. A. Meguid, "Multiscale modeling of the nonlinear response of nano-reinforced polymers," Acta Mechanica, vol. 217, no. 1-2, pp. 1-16, 2011.

[32] L. S. Schadler, S. C. Giannaris, and P. M. Ajayan, "Load transfer in carbon nanotube epoxy composites," Applied Physics Letters, vol. 73, no. 26, pp. 3842-3844, 1998.

[33] H. Wagner, "Nanotube-polymer adhesion: a mechanics approach," Chemical Physics Letters, vol. 361, no. 1-2, pp. 57-61, 2002.

[34] P. Ajayan, L. Schadler, C. Giannaris, and A. Rubio, "Singlewalled carbon nanotube-polymer composites: strength and weakness," Advanced Materials, vol. 12, no. 10, pp. 750-753, 2000.

[35] C. A. Cooper, R. J. Young, and M. Halsall, "Investigation into the deformation of carbon nanotubes and their composites through the use of Raman spectroscopy," Composites A, vol. 32, no. 3-4, pp. 401-411, 2001.

[36] V. Hadjiev, M. Iliev, S. Arepalli, P. Nikolaev, and B. S. Files, "Raman scattering test of single-wall carbon nanotube composites," Applied Physics Letters, vol. 78, no. 21, pp. 3193-3195, 2001.

[37] A. Paipetis, C. Galiotis, Y. C. Liu, and J. A. Nairn, "Stress transfer from the matrix to the fibre in a fragmentation test: Raman experiments and analytical modeling," Journal of Composite Materials, vol. 33, no. 4, pp. 377-399, 1999.

[38] L. Valentini, J. Biagiotti, J. M. Kenny, and M. A. López Manchado, "Physical and mechanical behavior of single-walled carbon nanotube/polypropylene/ethylene-propylene-diene rubber nanocomposites," Journal of Applied Polymer Science, vol. 89, no. 10, pp. 2657-2663, 2003.

[39] D. Qian, G. J. Wagner, W. K. Liu, M. Yu, and R. S. Ruoff, "Mechanics of carbon nanotubes," Applied Mechanics Reviews, vol. 55, no. 6, pp. 495-532, 2002.

[40] D. Qian, W. K. Liu, and R. S. Ruoff, "Load transfer mechanism in carbon nanotube ropes," Composites Science and Technology, vol. 63, no. 11, pp. 1561-1569, 2003.

[41] M. Rahmat and P. Hubert, "Carbon nanotube-polymer interactions in nanocomposites: a review," Composites Science and Technology, vol. 72, no. 1, pp. 72-84, 2011.

[42] A. V. Desai and M. A. Haque, "Mechanics of the interface for carbon nanotube-polymer composites," Thin-Walled Structures, vol. 43, no. 11, pp. 1787-1803, 2005.

[43] W. F. van Gunsteren and H. J. C. Berendsen, "Computer simulation of molecular dynamics: methodology, applications, and 
perspectives in chemistry," Angewandte Chemie, vol. 29, no. 9, pp. 992-1023, 1990.

[44] D. C. Rapaport, The Art of Molecular Dynamics Simulation, Cambridge University Press, Cambridge, UK, 2004.

[45] V. Kalra and Y. L. Joo, "Coarse-grained molecular dynamics study of block copolymer/nanoparticle composites under elongational flow," Journal of Chemical Physics, vol. 131, no. 21, Article ID 214904, 2009.

[46] M. Rahmat, H. Ghiasi, and P. Hubert, "Interaction energy and polymer density profile in nanocomposites: a coarse grain simulation based on interaction stress," Polymer Chemistry, vol. 3, no. 5, pp. 1158-1167, 2012.

[47] S. C. Chowdhury and T. Okabe, "Computer simulation of carbon nanotube pull-out from polymer by the molecular dynamics method," Composites $A$, vol. 38 , no. 3, pp. 747-754, 2007.

[48] Y. Kuang and X. He, "Young's moduli of functionalized singlewall carbon nanotubes under tensile loading," Composites Science and Technology, vol. 69, no. 2, pp. 169-175, 2009.

[49] J.-L. Tsai, S.-H. Tzeng, and Y.-T. Chiu, "Characterizing elastic properties of carbon nanotubes/polyimide nanocomposites using multi-scale simulation," Composites B, vol. 41, no. 1, pp. 106-115, 2010.

[50] G. Odegard, T. Gates, K. Wise, C. Park, and E. J. Siochi, “Constitutive modeling of nanotube-reinforced polymer composites," Composites Science and Technology, vol. 63, no. 11, pp. 1671-1687, 2003.

[51] S. J. V. Frankland and V. M. Harik, "Analysis of carbon nanotube pull-out from a polymer matrix," Surface Science, vol. 525, no. 13, pp. L103-L108, 2003.

[52] M. Al-Haik, M. Y. Hussaini, and H. Garmestani, "Adhesion energy in carbon nanotube-polyethylene composite: effect of chirality," Journal of Applied Physics, vol. 97, no. 7, Article ID 074306, 2005.

[53] R. E. Rudd, "Coarse-grained molecular dynamics for computer modeling of nanomechanical systems," International Journal for Multiscale Computational Engineering, vol. 2, no. 2, p. 157, 2004.

[54] S. Cranford and M. J. Buehler, "Coarse-graining parameterization and multiscale simulation of hierarchical systems: part I: theory and model formulation," in Multiscale Modeling: From Atoms to Devices, P. Derosa and T. Cagin, Eds., Taylor \& Francis, London, UK, 2010.

[55] P. Derreumaux and N. Mousseau, "Coarse-grained protein molecular dynamics simulations," Journal of Chemical Physics, vol. 126, no. 2, Article ID 025101, 2007.

[56] N. K. Vaidya, H. Huang, and S. Takagi, "Coarse grained molecular dynamics simulation of interaction between hemagglutinin fusion peptides and lipid bilayer membranes," Advances in Applied Mathematics and Mechanics, vol. 2, pp. 430-450, 2010.

[57] M. Zhang and F. Müller-Plathe, "The Soret effect in dilute polymer solutions: influence of chain length, chain stiffness, and solvent quality," Journal of Chemical Physics, vol. 125, no. 12, Article ID 124903, 2006.

[58] A. Di Matteo, F. Müller-Plathe, and G. Milano, "From mesoscale back to atomistic models: a fast reverse-mapping procedure for vinyl polymer chains," Journal of Physical Chemistry B, vol. 111, no. 11, pp. 2765-2773, 2007.

[59] T. Terao, E. Lussetti, and F. M. Müller-Plathe, "Non-equilibrium molecular dynamics methods for computing the thermal conductivity: application to amorphous polymers," Physical Review E, vol. 75, no. 5, Article ID 057701, 2007.
[60] M. Fermeglia and S. Pricl, "Multiscale modeling for polymer systems of industrial interest," Progress in Organic Coatings, vol. 58, no. 2-3, pp. 187-199, 2007.

[61] C. D. Wick and D. N. Theodorou, "Connectivity-altering Monte Carlo simulations of the end group effects on volumetric properties for poly(ethylene oxide)," Macromolecules, vol. 37, no. 18, pp. 7026-7033, 2004.

[62] V. A. Harmandaris, N. P. Adhikari, N. F. A. Van Der Vegt, and K. Kremer, "Hierarchical modeling of polystyrene: from atomistic to coarse-grained simulations," Macromolecules, vol. 39, no. 19, pp. 6708-6719, 2006.

[63] V. A. Harmandaris, D. Reith, N. F. A. Van Der Vegt, and K. Kremer, "Comparison between coarse-graining models for polymer systems: two mapping schemes for polystyrene," Macromolecular Chemistry and Physics, vol. 208, no. 19-20, pp. 2109-2120, 2007.

[64] S. Nielsen, C. F. Lopez, G. Srinivas, and M. L. Klein, "A coarse grain model for $\mathrm{n}$-alkanes parameterized from surface tension data," Journal of Chemical Physics, vol. 119, no. 14, pp. 7043-7049, 2003.

[65] J. Zhao, S. Nagao, and Z. Zhang, "Thermomechanical properties dependence on chain length In bulk polyethylene: coarsegrained molecular dynamics simulations," Journal of Materials Research, vol. 25, no. 3, pp. 537-544, 2010.

[66] J. Zhao, S. Nagao, Z. L. Zhang, and H. Kristiansen, "Coarsegrained molecular dynamics simulations on size effect of glassy polyethylene particles," Journal of Nanoscience and Nanotechnology, vol. 10, no. 11, pp. 7340-7342, 2010.

[67] S. W. Cranford and M. J. Buehler, "In silico assembly and nanomechanical characterization of carbon nanotube buckypaper," Nanotechnology, vol. 21, no. 26, Article ID 265706, 2010.

[68] B. Xie, Y. Liu, Y. Ding, Q. Zheng, and Z. Xu, "Mechanics of carbon nanotube networks: microstructural evolution and optimal design," Soft Matter, vol. 7, no. 21, pp. 10039-10047, 2011.

[69] Y. Li and M. Kröger, "Viscoelasticity of carbon nanotube buckypaper: zipping-unzipping mechanism and entanglement effects," Soft Matter, vol. 8, pp. 7822-7830, 2012.

[70] G. Kim, Y. Kim, and J. Ihm, "Encapsulation and polymerization of acetylene molecules inside a carbon nanotube," Chemical Physics Letters, vol. 415, no. 4-6, pp. 279-282, 2005.

[71] K. Mylvaganam and L. C. Zhang, "Nanotube functionalization and polymer grafting: an ab initio study," Journal of Physical Chemistry B, vol. 108, no. 39, pp. 15009-15012, 2004. 

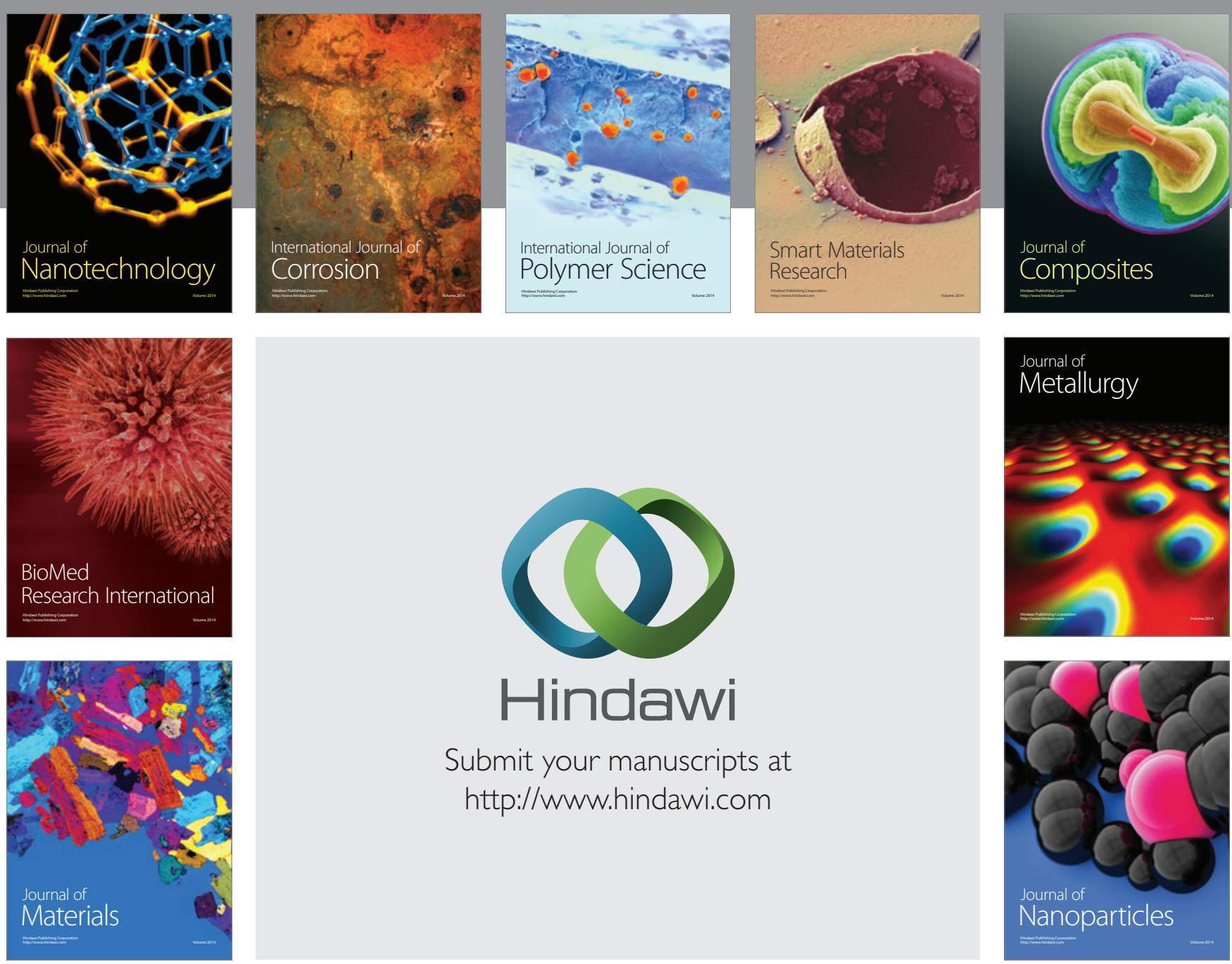

Submit your manuscripts at http://www.hindawi.com
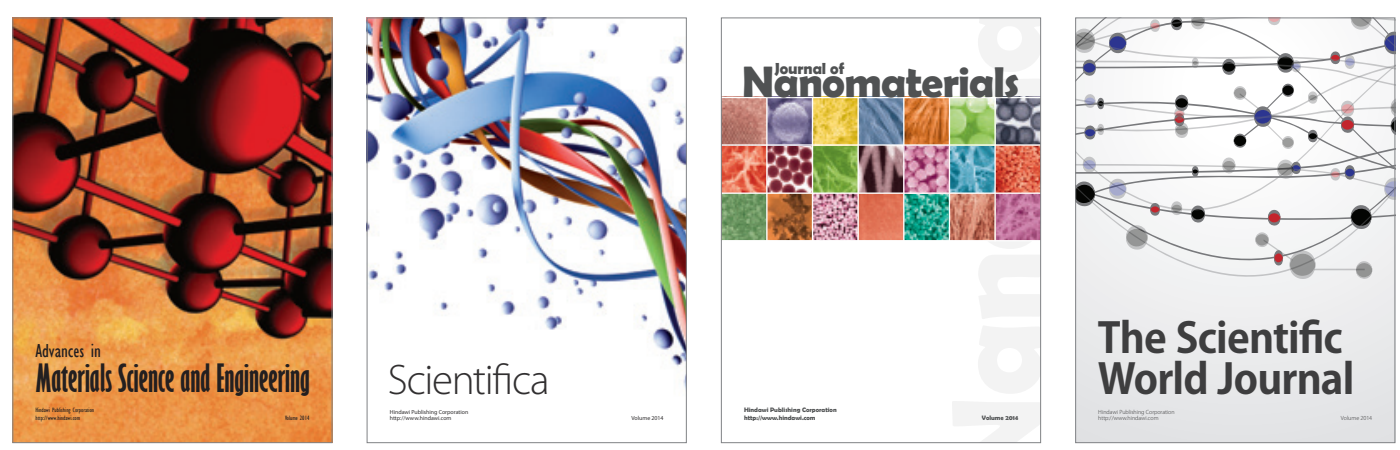

\section{The Scientific World Journal}
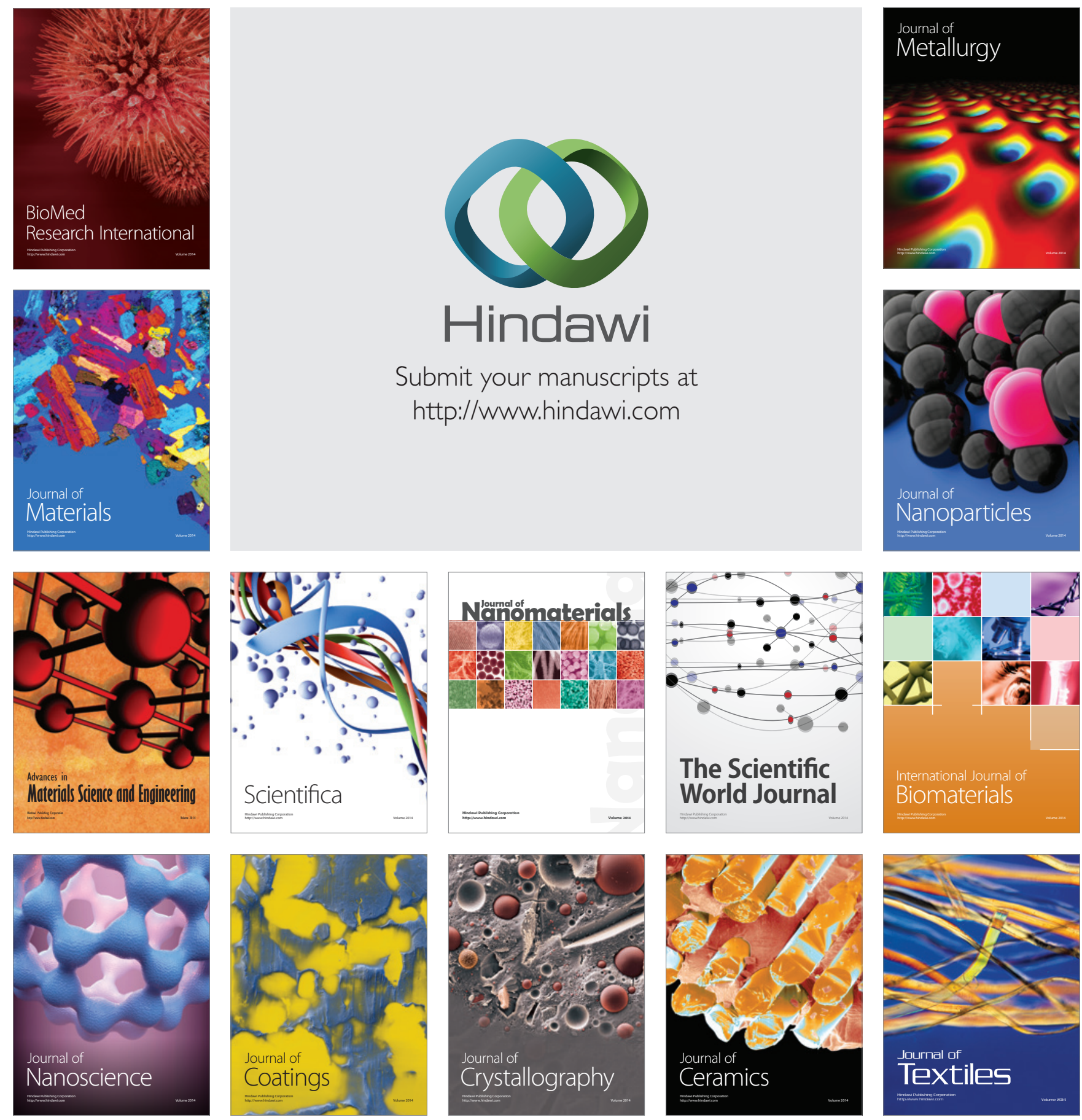